\title{
Simultaneous Bilateral Laparoscopic Adrenalectomy for Adrenal Metastases of Renal Cell Carcinoma: A Case Report
}

\author{
Akbar Nouralizadeh, MD, Amir Afyouni, MD, Behnam Shakiba, MD, and Fahad Khalid Radhi, MD ${ }^{1,2}$
}

\begin{abstract}
The possibility of bilateral adrenal gland metastasis from renal cell carcinoma (RCC) has been extremely rarely reported. Surgery may be the preferred treatment strategy for adrenal metastasis from RCC. In this article, we present a patient with bilateral large adrenal metastases from the left RCC, which was managed with simultaneous bilateral laparoscopic excision. A 64-year-old woman had undergone left adrenal sparing radical nephrectomy for a left RCC 7 years ago. Her abdominal CT scan revealed bilateral large adrenal metastasis. She underwent bilateral laparoscopic adrenalectomy. The operation was uneventful and the patient was discharged after 4 days with no complications. Bilateral laparoscopic adrenalectomy for adrenal metastasis seems to be a feasible and safe procedure in such cases, but this needs to be confirmed by further studies with a larger sample size.
\end{abstract}

Keywords: laparoscopy, adrenalectomy, adrenal glands, neoplasm metastasis, renal cell carcinoma

\section{Introduction}

$\mathbf{R}$ enal Cell Carcinoma (RCC) represents $\sim 3 \%$ of all types of cancer worldwide, and accounts for $90 \%$ to 95\% of malignancies involving the kidneys. ${ }^{1}$ Although, RCC metastasis can develop in every organ, the most common sites for distant metastasis are the lungs, liver, bones, and brain. $^{2}$ The risk of adrenal metastasis is low; consequently, most patients undergo adrenal sparing radical nephrectomy. The incidence of ipsilateral metastasis in the adrenal gland has been reported to be $3 \%-5 \%$, whereas the rate of contralateral metastasis is $0.7 \%$. The possibility of synchronous bilateral adrenal gland metastasis has been recorded as extremely rare $(<0.5 \%){ }^{3-5}$ Nevertheless, surgical resection of metastases from RCC remains the only therapeutic option in patients with a solitary site or low-volume metastasis. Therefore, surgery may be the preferred treatment strategy for adrenal metastasis from RCC. ${ }^{6}$ In this article, we present a patient with bilateral large adrenal metastases from the left RCC, which was managed with simultaneous bilateral laparoscopic excision.

\section{Case Report}

A 64-year-old Iranian woman was referred to our clinic; she had undergone left adrenal sparing radical nephrectomy for a left RCC, 7 years before. Microscopic histopathologic study showed a clear cell type of RCC. Abdominal CT scan revealed a right adrenal mass measuring $41 \times 33 \mathrm{~mm}$. In addition, two other masses measuring $40 \times 33$ and $30 \times 24 \mathrm{~mm}$ were detected in the left adrenal (Fig. 1). No calcification and contrast enhancement were observed in these masses.

The patient was admitted for further investigation. On physical examination, the patient had normal blood pressure and no other abnormalities were noted. The results of laboratory studies, including blood cells count, blood chemistry, and electrolytes, were within normal limits. Hormonal examination, including adrenocorticotropic hormone, serum catecholamines, cortisol, aldosterone, 24-hour urinary excretion of metanephrines and vanillylmandelic acid, and plasma renin activities were all within normal limits. Left adrenal CT-guided needle biopsy was then performed and the Iran.

${ }^{1}$ Urology and Nephrology Research Center, Shahid Labbafinejad Hospital, Shahid Beheshti University of Medical Sciences, Tehran,

${ }^{2}$ Department of Urology, Aljomhori General Hospital, Basrah, Iraq.

(c) Akbar Nouralizadeh et al. 2017; Published by Mary Ann Liebert, Inc. This is an Open Access article distributed under the terms of the Creative Commons Attribution License, which permits unrestricted use, distribution, and reproduction in any medium, provided the original work is properly cited. 


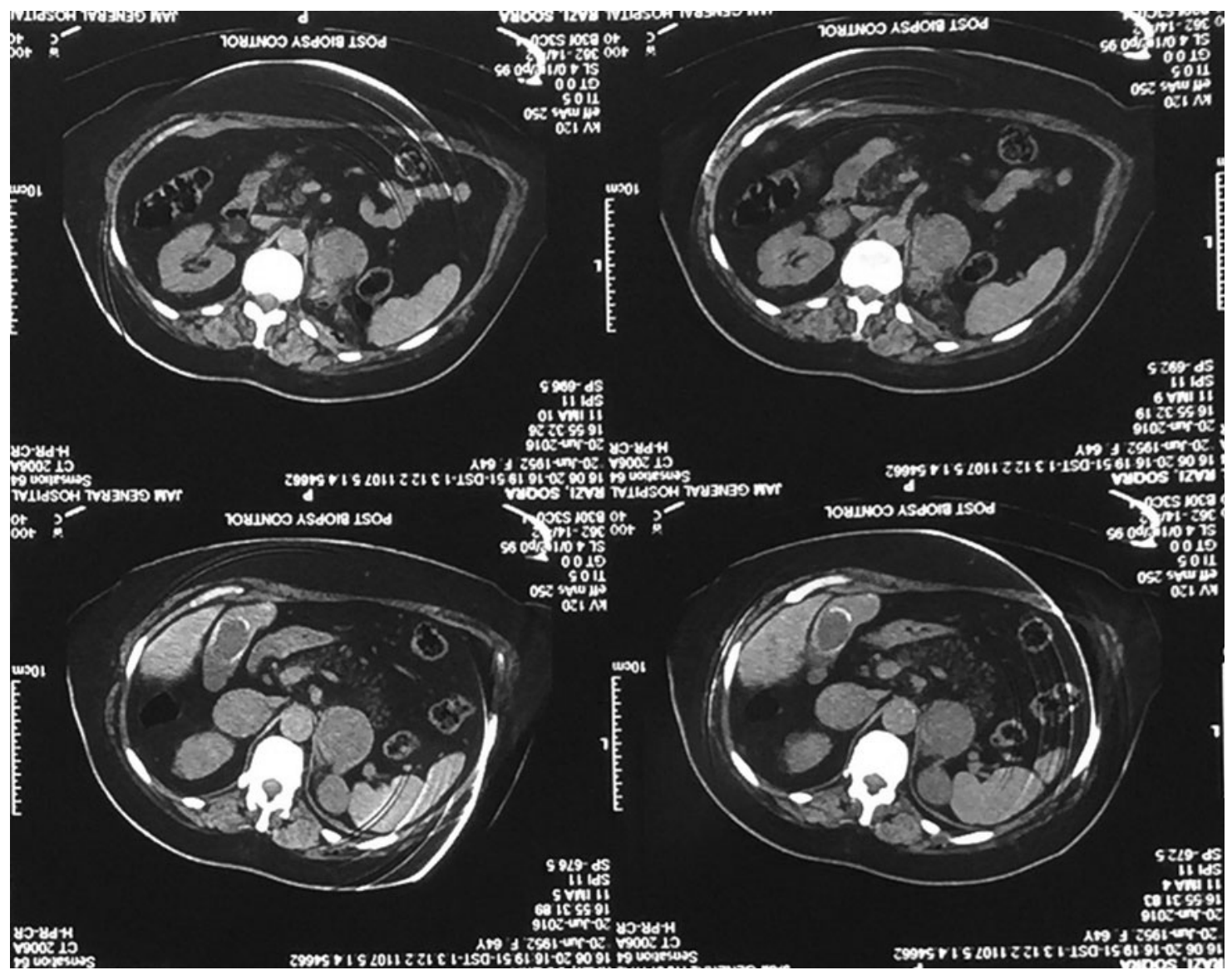

FIG. 1. Abdominal CT-identified bilateral adrenal masses.

histopathologic assessment of the biopsy sample revealed RCC metastasis.

She underwent bilateral laparoscopic adrenalectomy. Laparoscopy was done by the transperitoneal lateral decubitus approach, as it best exposes the gland and vessels. We used the Hasson technique to create pneumoperitoneum and the operation was done by four working trocars.

For the left side, it was necessary to mobilize the colon and tail of the pancreas. After dividing the splenocolic ligament and opening the Gerota fascia, the adrenal tumor was adequately exposed. The adrenal mass was reflected medially and the adrenal vein was clipped and divided. After extraction of the left adrenal gland, the right adrenal gland was excised effectively with the same approach (Fig. 2). The operation was uneventful and the patient was discharged after 4 days with no complications. Microscopic histopathology showed bilateral adrenal metastases of RCC.

\section{Discussion}

Synchronous ipsilateral adrenalectomy is not routinely recommended in radical nephrectomy as the risk of ipsilateral adrenal gland involvement is around $2 \%{ }^{4}$ Furthermore, adrenal gland metastasis may be discovered many years after radical nephrectomy. ${ }^{2}$ The mean time required to develop a metastasis from a RCC to the contralateral adrenal gland has been identified as 52.3 months after radical nephrectomy. ${ }^{4}$ The incidence of ipsilateral, contralateral, and bilateral adrenal metastasis from RCC are $1.9 \%, 1.5 \%$, and $0.3 \%$, respectively. ${ }^{7}$

Given all that, certain problems have to be overcome in the diagnosis of adrenal metastasis. First, the clinical signs and symptoms of adrenal metastasis are rare. Nowadays, patients'

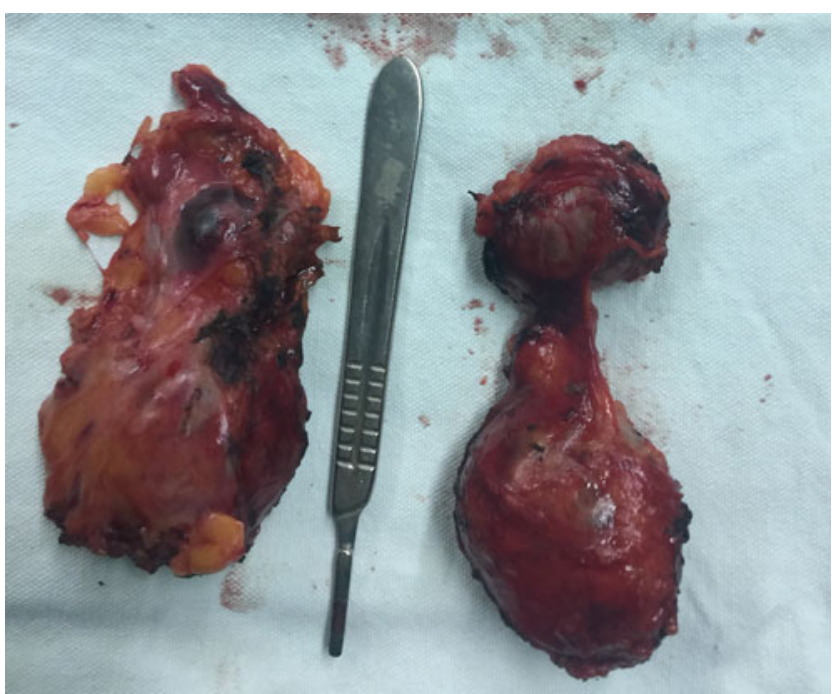

FIG. 2. Bilateral adrenal glands were extracted. 
follow-up with routine noninvasive radiologic techniques, such as CT, have improved the detection of adrenal metastatic lesions. In some patients, failure to use routine imaging studies in the follow-up period might result in delayed diagnosis. Second, the distinction between metastatic lesions, primary adrenal malignancy, and adrenal adenoma may be problematic. The history of RCC, radiologic findings and normal hormonal assessment could be suggestive of a metastatic lesion involving the adrenal. In doubtful cases, needle biopsy may be helpful in the diagnosis, but the definite diagnosis is often made only by the histopathologic study following adrenalectomy. ${ }^{8}$

To date, various therapeutic modalities, including chemotherapy, hormonal therapy, and radiotherapy have failed to markedly improve survival in metastatic cases. Previous studies have shown that adrenalectomy with the aim of removing metastatic lesions may prolong survival in patients with adrenal metastasis. ${ }^{8}$ After adrenalectomy, the 3-year survival rate increases from $35 \%$ to $60 \%$, while the 5-year survival increases from $14 \%$ to $38 \% .^{2}$

Reoperation for open adrenalectomy after ipsilateral nephrectomy has been associated with some difficulties and a high rate of surgical complications. Abel et al. reported minimal morbidity and complications in eight patients having undergone laparoscopic adrenalectomy. ${ }^{6}$ In addition, laparoscopic adrenalectomy has some advantages such as rapid recovery time, less pain, better cosmetic results, lower percentage of incisional hernia, and rapid return to possible systemic therapy for metastatic diseases. ${ }^{2}$

Therefore, laparoscopic adrenalectomy is the preferred treatment for adrenal gland metastasis in RCC patients; however, experience with simultaneous laparoscopic bilateral adrenalectomy is limited. Some articles have reported simultaneous laparoscopic bilateral adrenalectomy for patients with bilateral adrenal disease. Takata et al. ${ }^{9}$ reported their results on simultaneous laparoscopic bilateral adrenalectomy in 30 patients with Cushing's disease and bilateral pheochromocytoma. They concluded that this procedure is safe and feasible. Kawasaki et al. ${ }^{10}$ also published their findings on 21 patients who underwent bilateral laparoscopic adrenalectomy for Cushing's disease, bilateral pheochromocytoma, aldosterone-producing adenoma, and bilateral metastasis of RCC. They reported a small number of minor perioperative complications, which proved the safety and efficacy of this procedure. In another report, Castillo et al. ${ }^{11}$ performed simultaneous laparoscopic bilateral adrenalectomy in 20 patients with bilateral pheochromocytoma, Cushing's disease, metastasis, congenital adrenal hyperplasia, hyperaldosteronism, adrenal adenoma, and myelolipoma. In their report, the sources of metastasis were lung cancer, melanoma, and lymphoma. Hasan et al. ${ }^{12}$ also reported the outcome of simultaneous laparoscopic bilateral adrenalectomy in seven patients with bilateral pheochromocytoma, Cushing's disease, and metastasis from lung cancer.

To the authors' knowledge, Kawasaki reported the first case of laparoscopic bilateral adrenalectomy for RCC metastasis, and therefore, the available literature on this procedure in RCC metastasis is limited.

Although the present study is a case report which is normally classified as low level evidence, yet, it shows that bilateral laparoscopic adrenalectomy for adrenal metastasis is a feasible approach. Some reports have stated that the laparoscopic approach is associated with a low rate of intraand postoperative complications, while preserving the principles of oncologic outcomes. ${ }^{10,11}$ Other studies have reported a higher complication rate in bilateral laparoscopic adrenalectomy compared with unilateral laparoscopic adrenalectomy; however, in the laparoscopic approach compared with the open one, there are less surgical complications and intraoperative risks. ${ }^{13}$ Moreover, extensive experience is required in advanced laparoscopic surgeries to perform a laparoscopic excision on malignant adrenal tumors and metastasis.

In conclusion, we presented a case of bilateral adrenal metastasis from left RCC, occurring 7 years after radical adrenal sparing nephrectomy. Laparoscopic bilateral adrenalectomy was effectively performed with no complications; yet, this approach needs to be confirmed by future studies and more cases.

\section{Disclosure Statement}

No competing financial interests exist.

\section{References}

1. Curti BD. Renal cell carcinoma. JAMA 2004;292:97-100.

2. Zografos GN, Farfaras A, Aggeli C, et al. Laparoscopic adrenalectomy for large adrenal metastasis from contralateral renal cell carcinoma. JSLS 2007;11:261-265.

3. Öztürk H. Bilateral synchronous adrenal metastases of renal cell carcinoma: A case report and review of the literature. Oncol Lett 2015;9:1897-1901.

4. Peters I, Hora M, Herrmann TR, et al. Incidence of synchronous and metachronous adrenal metastases following tumor nephrectomy in renal cell cancer patients: A retrospective bi-center analysis. Springerplus 2013;2:293.

5. Moslemi MK, Saghafi H, Firoozabadi MH. Renal cell carcinoma with simultaneous bilateral adrenal metastasis: Ipsilateral radical nephrectomy with contralateral adrenal preservation. Case Rep Oncol 2010;3:372-379.

6. Abel EJ, Karam JA, Carrasco A, et al. Laparoscopic adrenalectomy for metachronous metastases after ipsilateral nephrectomy for renal-cell carcinoma. J Endourol 2011;25: 1323-1327.

7. Antonelli A, Cozzoli A, Simeone C, et al. CoscianiCunico S. Surgical treatment of adrenal metastasis from renal cell carcinoma: A single-centre experience of 45 patients. BJU Int 2006;97:505-508.

8. Lau WK, Zincke H, Lohse CM, et al. Contralateral adrenal metastasis of renal cell carcinoma: Treatment, outcome and a review. BJU Int 2003;91:775-779.

9. Takata $\mathrm{MC}$, Kebebew E, Clark $\mathrm{OH}$, et al. Laparoscopic bilateral adrenalectomy: Results for 30 consecutive cases. Surg Endosc 2008;22:202-207.

10. Kawasaki Y, Ishidoya S, Kaiho Y, et al. Laparoscopic simultaneous bilateral adrenalectomy: Assessment of feasibility and potential indications. Int J Urol 2011;18: 762-767.

11. Castillo OA, Vitagliano G, Cortes O, et al. Bilateral laparoscopic adrenalectomy. J Endourol 2007;21:1053-1058.

12. Hasan R, Harold KL, Matthews BD, et al. Outcomes for laparoscopic bilateral adrenalectomy. J Laparoendosc Adv Surg Tech A 2002;12:233-236.

13. Yadav K, Bakshi G, Prakash G, et al. Simultaneous bilateral laparoscopic adrenalectomy for pheochromocytoma in 
multiple endocrine neoplasia (MEN) syndrome: Case report with review literature. Int J Surg Case Rep 2014;5: 487-490.

Address correspondence to: Behnam Shakiba, MD Urology and Nephrology Research Center Shahid Labbafinejad Hospital Shahid Beheshti University of Medical Sciences 9th Bostan Street, Pasdaran Street Tehran 1666679951

Iran

E-mail: behkiba@gmail.com; b_shakiba@razi.tums.ac.ir

\begin{tabular}{|c|}
\hline $\begin{array}{c}\text { Abbreviations Used } \\
\mathrm{CT}=\text { computed tomography } \\
\mathrm{RCC}=\text { renal cell carcinoma }\end{array}$ \\
$\begin{array}{l}\text { Cite this article as: Nouralizadeh A, Afyouni A, Shakiba } \\
\text { B, Radhi FK (2017) Simultaneous bilateral laparoscopic } \\
\text { adrenalectomy for adrenal metastases of renal cell } \\
\text { carcinoma: a case report, Journal of Endourology Case } \\
\text { Reports 3:1, 142-145, DOI: 10.1089/cren.2017.0062. }\end{array}$ \\
\hline
\end{tabular}

